\title{
The recurrence pattern following delayed breast reconstruction after mastectomy for breast cancer suggests a systemic effect of surgery on occult dormant micrometastases
}

\author{
Hanna Dillekås ${ }^{1,2} \cdot$ Romano Demicheli $^{3}$ - Ilaria Ardoino ${ }^{3}$ - Svein A. H. Jensen ${ }^{4}$. \\ Elia Biganzoli ${ }^{3} \cdot$ Oddbjørn Straume $^{1,5}$
}

Received: 13 May 2016/Accepted: 4 June 2016/Published online: 15 June 2016

(c) The Author(s) 2016. This article is published with open access at Springerlink.com

\begin{abstract}
The purpose of this study was to characterize the recurrence dynamics in breast cancer patients after delayed reconstruction. We hypothesized that surgical reconstruction might stimulate dormant micrometastases and reduce time to recurrence. All mastectomy breast cancer patients with delayed surgical reconstruction at Haukeland University Hospital, between 1977 and 2007, $n=312$, were studied. Our control group consisted of 1341 breast cancer patients without reconstruction. For each case, all patients in the control group with identical $\mathrm{T}$ and $\mathrm{N}$ stages and age \pm 2 years were considered. A paired control was randomly selected from this group. 10 years after primary surgery, 39 of the cases had relapsed, compared to 52 of the matched controls. The reconstructed group was analyzed for relapse dynamics after mastectomy; the first peak in relapses was similarly timed, but smaller than for the controls, while the second peak was similar in time and size. Second, the relapse pattern was analyzed with reconstruction as the starting point. A peak in recurrences
\end{abstract}

Hanna Dillekås, Romano Demicheli, Elia Biganzoli, and Oddbjørn Straume have contributed equally to this work.

Oddbjørn Straume

oddbjorn.straume@helse-bergen.no

1 Department of Oncology, Haukeland University Hospital, 5021 Bergen, Norway

2 Department of Clinical Science, University of Bergen, 5012 Bergen, Norway

3 Scientific Directorate, Fondazione IRCCS Istituto Nazionale Tumori, 20133 Milan, Italy

4 Department of Plastic- and Reconstructive Surgery, Haukeland University Hospital, 5021 Bergen, Norway

5 Centre of Cancer Biomarkers, University of Bergen, 5012 Bergen, Norway was found after 18 months, and a lower peak at the 5th-6th year. The height of the peak correlated with the extent of surgery and initial $\mathrm{T}$ and $\mathrm{N}$ stages. Timing of the peak was not affected, neither was the cumulative effect. The relapse pattern, when time origin is placed both at mastectomy and at reconstruction, is bimodal with a peak position at the same time points, at 2 years and at 5-6 years. The timing of the transition from dormant micrometastases into clinically detectable macrometastases might be explained by an enhancing effect of surgery.

Keywords Breast cancer - Surgery - Breast reconstruction - Recurrence dynamics - Tumor dormancy . Multivariate regression

\section{Introduction}

The major cause of breast cancer mortality is metastatic disease, and the prevention of metastatic spread and growth is the aim of primary local and systemic therapy [1]. Still, after initial treatment with curative intent, breast cancer is known for its potential to cause late relapse. Even tiny tumors, undetectable by physical, biochemical, or radiological examination, can shed malignant cells into the circulation and eventually cause recurrences up to 20 years after the primary surgery [1]. The current view of breast cancer as a systemic disease at the time of diagnosis was introduced by Fisher in the late 1960s [2]. The concept of tumor dormancy has been proposed [3-6] as an explanation of the latency of metastatic disease, and the past and current research is beginning to unravel the mechanisms of maintenance as well as disruption of dormancy [7]. Early micrometastatic foci can be restricted in growth over periods of time by inability to recruit blood vessels [8], by 
immune surveillance $[9,10]$, by cell cycle arrest $[11]$, or by tumor-microenvironment (TME) interactions [12].

Signs of stimulation of micrometastases after surgical intervention have been observed in experimental and epidemiological studies and evaluated in mathematical models [13-15]. Furthermore, clinical investigations support an enhancing effect on the growth of metastases after surgical primary tumor removal $[16,17]$. Tissue trauma and subsequent wound healing have been shown to cause both local and systemic growth signaling cascades, and might thereby possibly alter the dormant state of occult micrometastases $[18,19]$. In a previous study, our findings indicated the presence of synchronized tumor growth in metastatic breast cancer [20]. In some reports on human cancers such as primary breast cancer [21], ovarian [22], colorectal [23], lymphoma [24], and others, tissue trauma has been associated with tumor progression. It was proposed that the primary surgery by itself can represent a stimulating event responsible for the peak in the incidence of metastatic disease observed around 2 years postoperatively independent of tumor stage [25-27]. In light of these findings, questions have been raised regarding the safety of delayed reconstructive surgery. Both increased and reduced risk of recurrence was reported after delayed breast reconstruction $[28,29]$. The aim of the present study was to characterize the recurrence dynamics in breast cancer patients that underwent delayed reconstructive surgery. We hypothesized that delayed reconstructive surgery might stimulate preexisting, occult dormant micrometastases and alter the recurrence dynamics.

\section{Patients and methods}

\section{Study population}

The study population for this retrospective analysis consists of all mastectomy breast cancer patients who underwent delayed reconstructive surgery at Haukeland University Hospital, Bergen, Norway, and had their primary treatment between 1977 and 2007. The respective reconstructive procedures were implant surgery, implants combined with flaps, deep inferior epigastric perforator (DIEP) flaps, and transverse rectus abdominis myocutaneous (TRAM) flaps. Distinction was not made between single- and multistage surgery. Altogether, 312 patients were included after exclusion of patients for whom both tumor size and nodal status were not known as well as patients with secondary, nonbreast cancers and DCIS (ductal carcinoma in situ) (Fig. 1). The hospital covers a population of 600,000 , and in this period, all late reconstructive surgical interventions following breast cancer in the region were performed here. Each patient's record was studied to validate diagnosis, patient and tumor characteristics, adjuvant therapy, time and type of reconstructive surgery, time of first recurrence, and recurrent site.

\section{Control group}

We received a control population from the Norwegian Cancer Registry comprising 1341 patients with breast cancer surgery in the same time period that had not undergone reconstructive surgery. Reporting breast cancer treatment to this registry is mandatory for all physicians in Norway, and the latest published evaluation from 2007 showed a $99 \%$ completeness of data [30]. For data quality purposes, patient's records were studied for validation of diagnosis, patient and tumor characteristics, adjuvant therapy, reconstructive surgery (excluded from the control group), time of first recurrence, and recurrent site in the same way as was done with the cases. Among the 1341 patients, a total of 473 patients were excluded (see Fig. 1 for details) leaving 868 patients, whose characteristics are shown in Table 1, which hereafter will be labeled "control group."

\section{Matching}

For each patient in the reconstruction group, all patients in the control group with identical $\mathrm{T}$ and $\mathrm{N}$ stages, age \pm 2 years, and follow-up without recurrence equal to or longer than the time to reconstruction of the respective matched reconstructed patient were considered. In this initial step, each case could have a number of candidate controls of 0-X. A reference day was calculated for each of the controls in these groups representing time from primary surgery for the control plus time from primary surgery until reconstruction for the matched case. Therefore, time from primary surgery until reconstruction/reference day could by calculated for cases and controls, respectively. A paired control was randomly selected from this group. If this group was empty, increased age interval up to 5 years was allowed as a first step, and in a few cases when the age difference was considered clinically relevant (e.g., pre- vs postmenopausal), patients with similar, but not identical $\mathrm{T}$ classification (e.g., T2 instead of T1) within the right age interval were considered. This group of 312 patients, whose characteristics are shown in Table 1, will be hereafter labeled "matched control group."

\section{Follow-up}

Time to recurrence (TTR) was recorded as the time from primary surgery to recurrence. The endpoint of primary interest was the first evidence of recurrence: survival times were calculated as the time elapsed since primary surgery 
Fig. 1 Inclusion and exclusion criteria employed to achieve case, control, and matched control populations. DCIS ductal carcinoma in situ, LCIS lobular carcinoma in situ, $B C T$ breast-conserving therapy. 1 Recurrence-free follow-up time equal to or longer than the time to reconstruction of the respective matched reconstructed patient

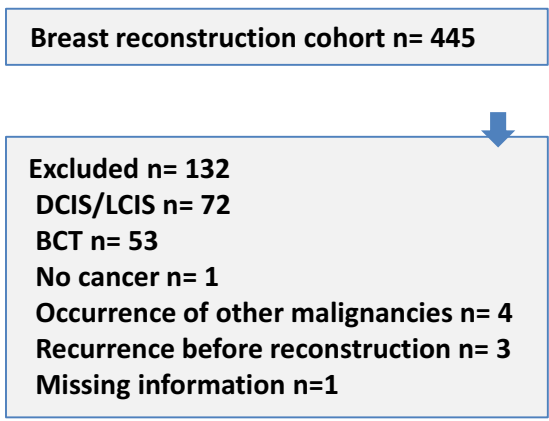

Control population from the Norwegian cancer registry $n=1341$.

Excluded $n=473$

DCIS/LCIS $n=37$

Reconstructed $\mathrm{n}=179$

BCT $n=196$

Missing information $\mathrm{n}=\mathbf{6 1}$

Control group $=868$

Matching on $\mathrm{T}, \mathrm{N}$, age, follow up free from recurrence ${ }^{1}$

Study population (reconstruction) $n=312$
Matched control group

(no reconstruction) $n=312$

Table 1 Patient, tumor, and treatment characteristics

\begin{tabular}{|c|c|c|c|}
\hline & $\begin{array}{l}\text { Reconstruction } \\
\text { group } n=312(\%)\end{array}$ & $\begin{array}{l}\text { Control group } \\
n=868(\%)\end{array}$ & $\begin{array}{l}\text { Matched control } \\
n=312(\%)\end{array}$ \\
\hline \multicolumn{4}{|l|}{ Year of primary diagnosis } \\
\hline $1977-1989$ & $15(4.8)$ & $57(6.6)$ & $25(8.0)$ \\
\hline 1990-1999 & $99(31.7)$ & $298(34.3)$ & $109(34.9)$ \\
\hline 2000-2009 & $198(63.5)$ & $513(59.1)$ & $178(57.1)$ \\
\hline Median age at diagnosis & 48.0 & 50.0 & 49.0 \\
\hline Mean age at diagnosis & 48.1 & 50.7 & 48.7 \\
\hline Age $<50$ & $171(54.8)$ & $397(45.7)$ & $171(54.8)$ \\
\hline Age $\geq 50$ & $141(45.2)$ & $471(54.3)$ & $141(45.2)$ \\
\hline \multicolumn{4}{|l|}{ Tumor size } \\
\hline $\mathrm{T} 1$ & $190(60.9)$ & $379(43.7)$ & $192(61.5)$ \\
\hline $\mathrm{T} 2$ & $91(29.2)$ & $332(38.2)$ & $94(30.1)$ \\
\hline $\mathrm{T} 3$ & $22(7.1)$ & $87(10.0)$ & $21(6.7)$ \\
\hline $\mathrm{T} 4$ & $2(0.6)$ & $43(5.0)$ & $2(0.6)$ \\
\hline Missing & $7(2.2)$ & $27(3.1)$ & $3(0.9)$ \\
\hline \multicolumn{4}{|l|}{ Nodes } \\
\hline Negative & $212(67.9)$ & $428(49.3)$ & $210(67.3)$ \\
\hline Positive & $100(32.1)$ & $421(48.5)$ & $102(32.7)$ \\
\hline Missing & & $19(2.2)$ & \\
\hline \multicolumn{4}{|l|}{ ER status } \\
\hline Negative & $61(19.6)$ & $190(21.9)$ & $60(19.2)$ \\
\hline Positive & $218(69.9)$ & $544(62.7)$ & $216(69.2)$ \\
\hline Missing & $33(10.6)$ & $134(15.4)$ & $36(11.5)$ \\
\hline \multicolumn{4}{|c|}{ Adjuvant endocrine treatment } \\
\hline No & $117(37.5)$ & $238(27.4)$ & $115(36.9)$ \\
\hline Yes & $136(43.6)$ & $379(43.7)$ & $132(42.3)$ \\
\hline Missing & $59(18.9)$ & $251(28.9)$ & $65(20.8)$ \\
\hline \multicolumn{4}{|l|}{ Adjuvant chemotherapy } \\
\hline No & $144(46.2)$ & $327(37.7)$ & $136(43.6)$ \\
\hline Yes & $143(45.8)$ & $305(35.2)$ & $125(40.1)$ \\
\hline Missing & $25(8.0)$ & $235(27.1)$ & $51(16.3)$ \\
\hline
\end{tabular}


to recurrence or to the last documented follow-up with no evidence of disease. Both locoregional recurrence and distant metastasis were defined as the events of interest, whereas all new primary tumors, including contralateral breast cancers, were considered competing events, thus for these patients survival times were censored at the time of their occurrence. Adjuvant local and/or systemic treatment was given according to national guidelines at the given time period and was not affected by delayed breast reconstruction. Follow-up after curative breast cancer treatment in Norway does not include radiologic evaluation or blood samples other than upon clinical suspicion of distant metastases. Thus, diagnosis of relapse is most commonly made after patients' experience of symptoms. Even when adopting more meticulous follow-up regimens, more than $85 \%$ of recurrences are detected following symptomatic alert and not at controls [31]. Oncological follow-up is not influenced by reconstructive surgery.

\section{Statistical analysis}

The event dynamics were studied by estimating with the life-table method the hazard rate for recurrence, i.e., the conditional probability of manifesting recurrence given that the patient is clinically free from any recurrence at the beginning of the interval. The probability of recurrence over time, i.e., crude cumulative incidence (CCI), was estimated according to a proper nonparametric estimator adjusting for the presence of competing events and compared by the Gray test [32]. A discretization of the time axis in six-month units was applied and a Kernel-like smoothing procedure [33] was adopted. For multivariable regression analysis, the piecewise exponential model was used. The piecewise exponential model provides a flexible semiparametric tool in the study of the hazard function for survival data, in the same fashion as a Cox regression model [34]. The log-hazard function was modeled as an additive function of the baseline log-hazard and the covariate effects. For estimation of the piecewise exponential model, the follow-up time was split into 3-month disjoint intervals and the event rate was assumed to be constant within each interval. The model accounts for reconstruction as a time-dependent covariate (i.e., switching from 0 to 1 at the time it was performed). The model was extended to account for the new timescale induced by reconstructive surgery, namely the time elapsed since reconstruction to the endpoint of interest [35]. For practical purposes, time since reconstruction assumed the value 0 , before its occurrence, as well as for the controls.

Available prognostic factors were taken into account to adjust the multivariable regression model. These included age at diagnosis, pathologic tumor size (T2-T4 vs. T1), nodal status (N+ vs. N0), and estrogen receptor status
$(\mathrm{ER}+$ vs. ER-), with time-dependent effect (by introducing Time (since primary tumor surgery) $*$ ER interaction). To allow for the estimation of baseline hazard, both timescales were modeled via Natural Splines with 5 knots (corresponding to the quantiles of event times only). For age at diagnosis, a possible nonlinear effect was also tested. Statistical analyses were done using R3.02 software for Windows, with Epi package added.

\section{Results}

Of the 312 patients, 302 had reconstructive surgery within 180 months and 291 within 120 months after primary surgery, whereas the remaining 10 had longer time to reconstruction. Median time to reconstruction was 33 months (range: 1-362 months). Median follow-up after reconstruction was 137 months. Within 10 years after primary surgery, 39 of the 312 reconstructed patients developed local (5), regional (6), or distant (31) relapse, compared to 52 patients in the matched control group (local 10, regional 3, distant 39).

As a first step, the recurrence dynamics for the reconstructed patients were analyzed with the time origin at primary cancer surgery (Fig. 2, blue line). As expected, a bimodal hazard rate pattern was observed, with an early less prominent peak in comparison with the second later one. When the recurrence dynamics were analyzed with reconstructive surgery as the time origin, a distinct early peak in recurrences was found around 18 months postreconstruction, followed by a second lower peak at the 5th6 th year (Fig. 2, red line). The height of this peak was dependent on the extent of surgery. More extensive surgeries like deep inferior epigastric perforator (DIEP) flaps,

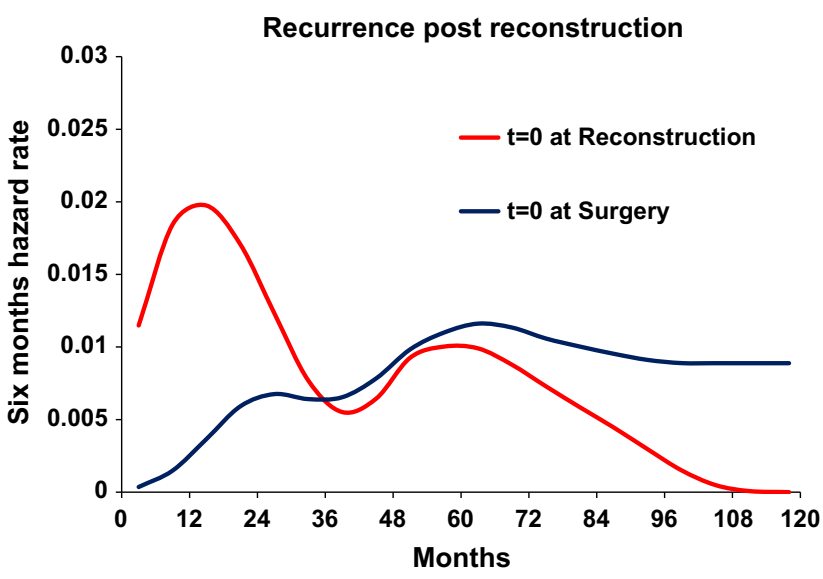

Fig. 2 Recurrence pattern for the reconstructed patients $(n=312)$ with $T=0$ set at reconstruction (red line) and at primary surgery (blue line). $X$-axis represents time in months. $Y$-axis represents sixmonth hazard rate 
transverse rectus abdominis myocutaneous (TRAM) flaps, and combined implant and flap surgery as compared with simple implant resulted in a higher peak for the former (Fig. 3). The timing of the peak was not affected by the extent of surgery. There was no difference in recurrencefree survival between extensive reconstructive surgery and simple implant surgery (Fig. 4, Gray test, $p=0.86$ ). Similarly, the height of the recurrence peaks, but not the timing, was dependent on known risk factors such as nodal involvement and $\mathrm{T}$ stage (Fig. 5).

The relapse pattern for the matched control group, when the time origin was set at mastectomy, followed the expected bimodal pattern with a first, dominant early peak and a second less marked peak at 5 years after primary surgery (Fig. 6), as frequently demonstrated in previous studies. When the time origin was moved to the reference day, the hazard rate curve appears as a simple distortion of the previous one (figure not reported). Unlike the reconstructed patients, no definite trait was detectable. The recurrence incidence was slightly reduced for the reconstructed patients in comparison with matched control patients, although the difference was not statistically significant (Fig. 7, Gray test, $p=0.08$ ).

The multiple scale analysis supports the hypothesis of a transitory significant increase of recurrence risk during the first two years after reconstruction/reference day for reconstructed patients in comparison with the not reconstructed patients. In the multivariable regression model, where all 868 patients in the control group were analyzed, all factors that were considered confirmed their expected prognostic impact, including the time-dependent effect of

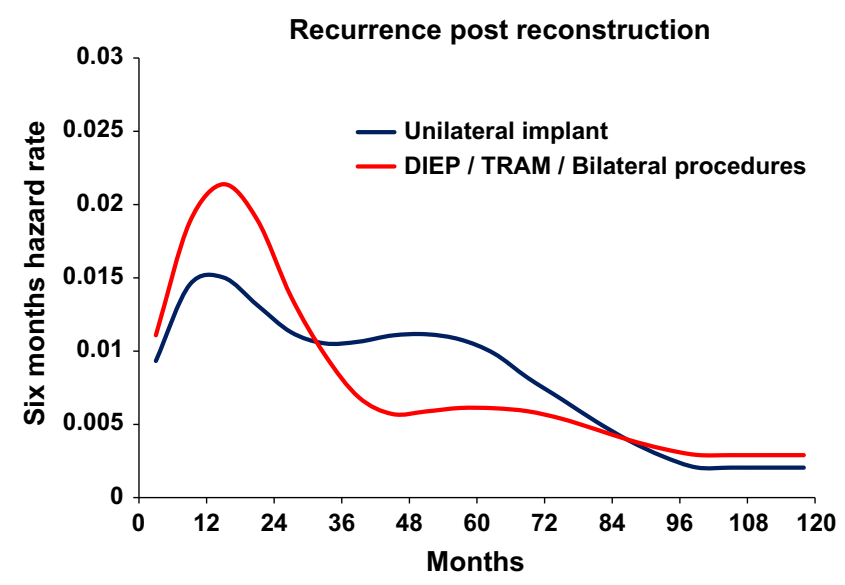

Fig. 3 Recurrence pattern according to surgical intervention demonstrates an enhanced, but similarly timed, effect by increased extent of trauma. Blue line patients receiving a unilateral implant. Red line patients receiving more extensive surgery. $X$-axis represents time in months since reconstructive surgery. $Y$-axis represents six-month hazard rate. DIEP deep inferior epigastric perforator, TRAM transverse rectus abdominis myocutaneous flap
ER status, whereas age at diagnosis did not. The hazard ratio (HR) was higher in node-positive patients and in those with increasing tumor size (Table 2). The multiple timescale model allows for understanding whether the time effect, induced by reconstruction occurrence, may be relevant for the subsequent risk of developing unfavorable events. To better interpret the model, a graph showing the effect of the timescale induced by reconstruction surgery is shown in Fig. 8. The figure shows how the hazard ratio for recurrence between reconstructed patients and control patients (with the same clinical and pathological features and with the same follow-up time since primary tumor surgery) may not be considered constant during the subsequent follow-up time. Although not fully significant with a moderately wide confidence interval, it shows an increased risk for the reconstructed patients within the first 2 years, with a peak at about 18 months after surgery and decreasing thereafter. Of note, the recurrence dynamics following reconstructive surgery were unaffected by the time from primary surgery to reconstruction, both in timing and magnitude.

\section{Discussion}

To our knowledge, this is the first study investigating the dynamics of recurrences occurring after delayed breast reconstruction in breast cancer patients. The main result of our analysis is that when the time origin is set at the reconstruction date, the hazard rate for ensuing recurrence displays a first main peak in the 2nd year and a later minor peak at the 5th-6th year after reconstruction (Fig. 2, red line). The recurrence risk for the same patients, when the time origin is placed at mastectomy, is bimodal with peak positions at the same time points relative to mastectomy, after 2 and 5-6 years (Fig. 2, blue line), as expected [31]. Thus, when the time origin is moved for each reconstructed patient to the reconstruction date, the recurrence risk pattern is similar to that observed following primary mastectomy. Of note, time origin displacement reveals an increase of the early peak with a concomitant decrease of the late level of recurrence risk (Fig. 2), suggesting that recurrence redistribution is associated with the reconstruction maneuver which could be said to act as a wave breaker for recurrences. These findings suggest that mastectomy and reconstruction induce similar biological effects on subclinical preexisting metastases.

The effects of primary mastectomy have been investigated in both animals and humans during the past century [36]. An unintentional effect of surgery in breast cancer patients with clinically undetectable micrometastatic disease has been explained by a paradigm based on the concepts of tumor homeostasis, tumor dormancy, and surgery- 
Fig. 4 The probability of recurrence over time, i.e., Crude Cumulative Incidence (CCI), proper nonparametric estimator adjusting for the presence of competing events and was compared between groups by the Gray test. Simple unilateral implant (black line) and more extensive surgery such as DIEP/ TRAM or bilateral procedures (red line). $X$-axis represents time since reconstruction in months. There is no observable difference between the groups was estimated according to

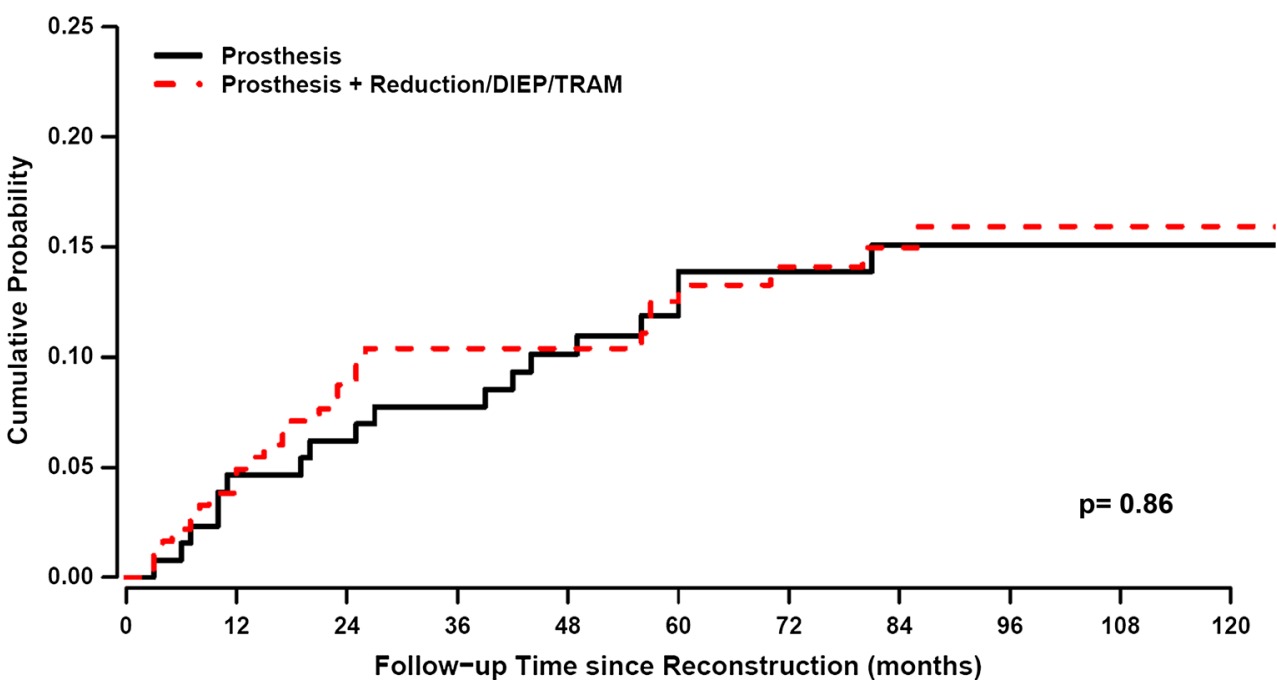

Recurrence post reconstruction

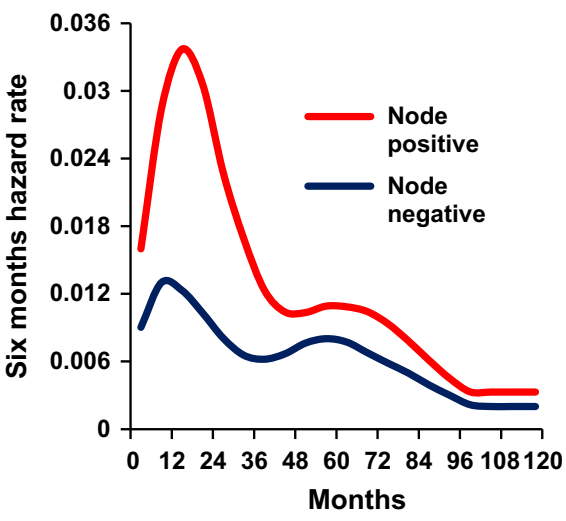

Fig. 5 Subgroup analysis of recurrence pattern by known prognostic factors. Increasing $\mathrm{T}$ and $\mathrm{N}$ stages are associated with an enhancing effect on preexisting recurrence risk. Left figure demonstrates the recurrence dynamics for node-positive (red line) and node-negative

related enhancement of metastasis growth [37]. The model assumes both cellular and micrometastatic tumor dormancy, with ordered transitions between these two quiescent states and subsequent development of overt metastasis and, subsequently, a transient phase of acceleration of metastatic growth. Preclinical studies have pointed to an angiogenic switch as a possible involved mechanism, in which the microenvironment is altered by tissue trauma to become proangiogenic with increased levels of VEGF and reduced levels of angiogenesis inhibitors such as TSP-1 [8]. Others have focused on the role of surgery-induced immunomodulation with demonstration of a stimulatory interaction between cells of the innate immune system and adjacent cancer cells [38]. The truth may lie in both models as the immune and angiogenic systems have multiple points of intersection [39]. Our findings support the concept that also delayed breast reconstruction may accelerate

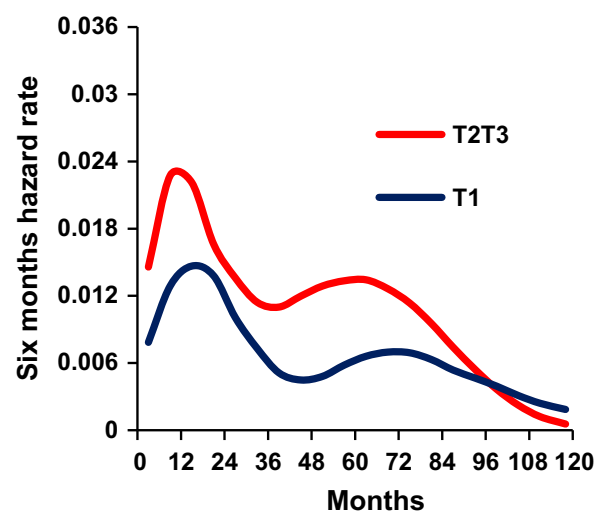

(blue line) reconstructed patients. Right figure demonstrates the recurrence dynamics for reconstructed patients with tumors $>2 \mathrm{~cm}$ (red line) and $\leq 2 \mathrm{~cm}$ (blue line). $X$-axis represents months since reconstruction. $Y$-axis represents six-month hazard rate

metastatic growth in subjects with dormant metastatic foci similar to the effects observed after primary surgery. This explanation is further supported by the finding that surgical approaches with different extents result in different recurrence risks, although with the same time rhythm (Fig. 3). Specifically, the more extensive reconstruction modalities DIEP/TRAM and bilateral surgical procedures give rise to a higher early peak in comparison with unilateral implant surgery. This difference is limited to the recurrence risk level, which is differently modulated within the same time cadence, while the two types of reconstruction do not affect long-term outcome differently (Fig. 4).

The proposed explanation assumes that the risk of clinical appearance of metastasis is dependent on the action of surgery-related factors on the subclinical metastatic state of the host. This assumption is confirmed by the marked influence of both tumor size and nodal status on the hazard 


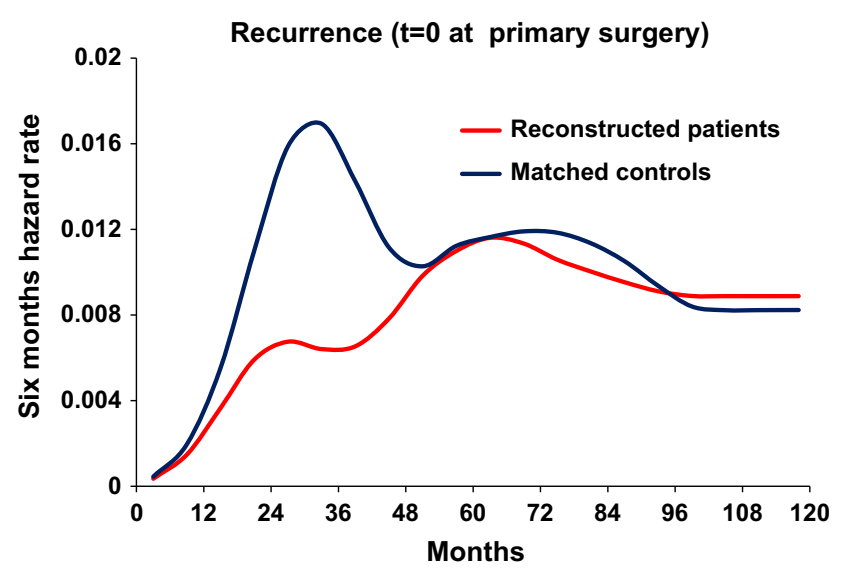

Fig. 6 Recurrence pattern for the reconstructed patients and the matched control patients with $T=0$ at primary surgery. Red line reconstructed patients. Blue line control patients. $X$-axis represents months since primary surgery. $Y$-axis represents six-month hazard rate

level (Fig. 5). At diagnosis, these two well-known prognostic factors indicate the recurrence risk during the disease course. Thus, it is coherent that an additional triggering factor, such as delayed surgical reconstruction, may result in different outcomes when patients with different underlying recurrence risk are involved.

When studying the relation between outcomes of patients undergoing delayed reconstruction in comparison with patients undergoing mastectomy without reconstruction, crucial problems emerge. In addition to the retrospective nature of such studies, the reconstructed group is
Table 2 Hazard ratio for recurrence according to tumor characteristics

\begin{tabular}{llll}
\hline & HR & 95\% CI & \\
\hline ER pos versus neg (18 months) & 0.30 & 0.19 & 0.47 \\
ER pos versus neg (60 months) & 1.77 & 0.81 & 3.86 \\
T2 versus T1 & 1.91 & 1.43 & 2.56 \\
T3 versus T1 & 2.78 & 1.89 & 4.09 \\
T4 versus T1 & 3.13 & 1.91 & 5.11 \\
$\mathrm{~N}+$ versus N- & 1.98 & 1.52 & 2.59 \\
\hline
\end{tabular}

characterized by a selection event not yet occurred (and therefore unknown) at the mastectomy time and, moreover, occurring at varying patient-related times during follow-up, thus raising important issues in the statistical analysis. To overcome these drawbacks, we used two different approaches. In the first approach, which has been frequently adopted in this field in spite of its intrinsic naivety $[28,29]$, we performed a matched random choice of the control patients. For each reconstructed patient, we randomly identified a matched control patient with similar initial characteristics, selecting her among the not reconstructed patients who were disease free at the date of reconstruction of the considered patient. This matching modality resulted in a good balance between the two sets of patients (Table 1) and avoided drawbacks detectable in published reports, such as dissimilar patient characteristics (e.g., age) [40] or dissimilar prognostic factors [28, 29]. In

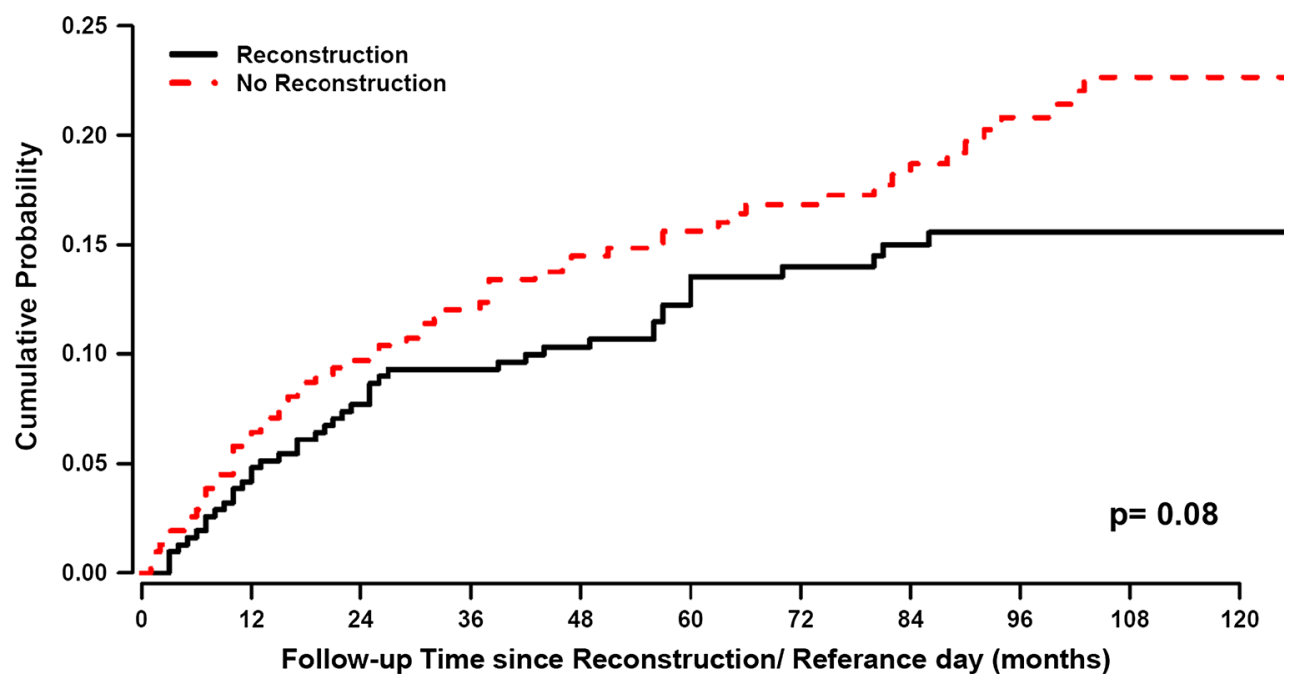

Fig. 7 The probability of recurrence over time, i.e., Crude Cumulative Incidence (CCI), was estimated according to proper nonparametric estimator adjusting for the presence of competing events and was compared between groups by the Gray test. Red line reconstructed patients. Blue line matched control patients. Despite matching by age, time of diagnosis, and $\mathrm{T}$ and $\mathrm{N}$ stage, there is a nonsignificant trend for a more favorable prognosis in the reconstructed patients. $X$-axis represents time in months since reconstruction/reference day (see $m \& m$ ) for reconstructed patients and control patients, respectively. $Y$-axis represents accumulated recurrence-free survival 


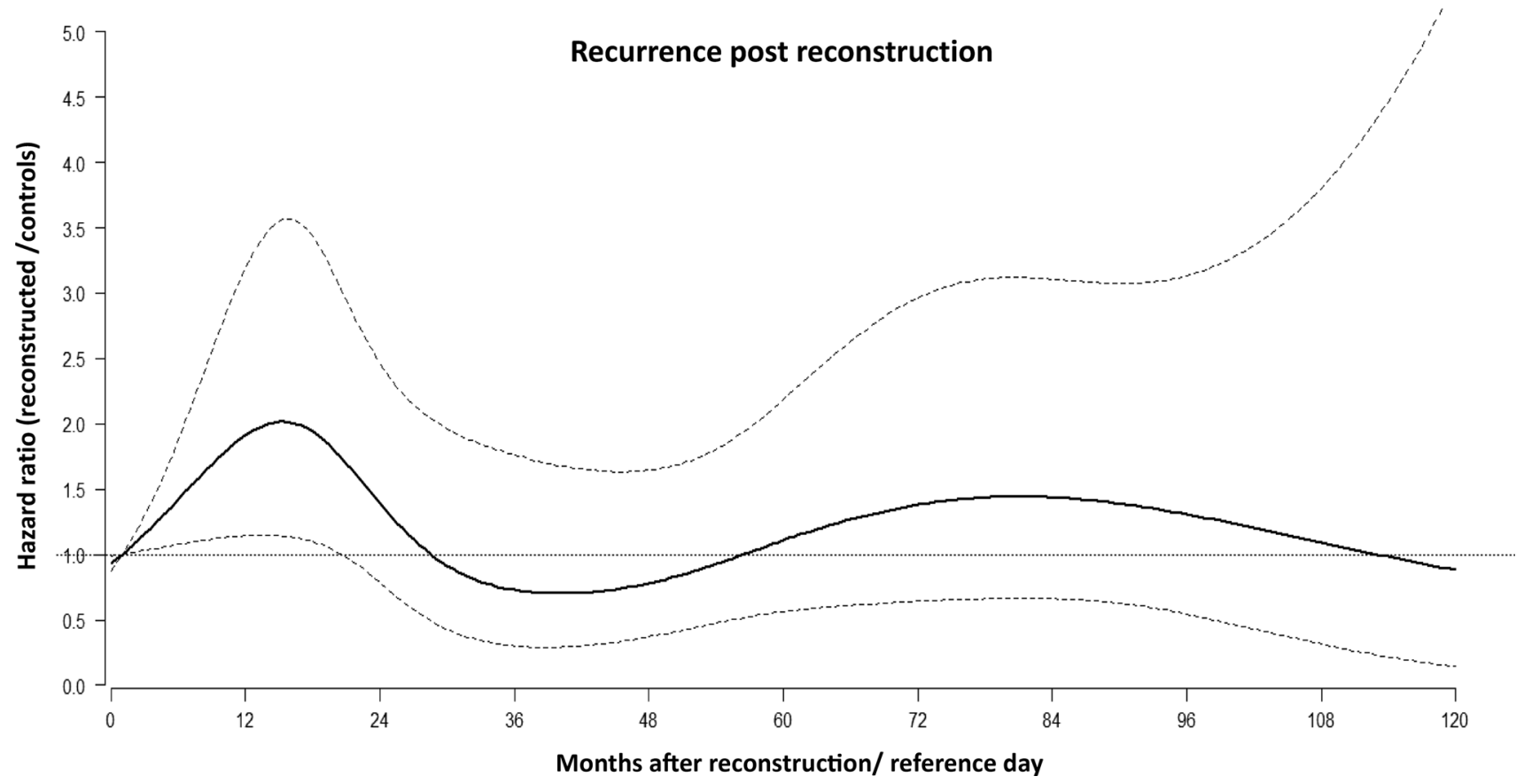

Fig. 8 Multiple timescale analysis of the hazard ratio for recurrence between the reconstructed patients and the controls in relation to time since reconstruction. Dotted lines represent $95 \%$ CI. $X$-axis

addition, the recurrence dynamics of matched controls, when time origin was set at mastectomy (Fig. 6, blue line), was coherent with the analogous hazard rate pattern observable in similar patients from different databases [31]. It displays a first peak significantly higher than the corresponding peak of reconstructed patients (Fig. 6, red line) revealing that, in spite of the balanced initial prognostic factors, matched control patients display worse prognosis than reconstructed patients. This finding is consistent with a population-based registry study analyzing Danish women who received reconstruction with implants only [41].

In the second approach, we addressed the analysis in the framework of multiple timescales in multistate models [35]. The regression analysis involved all 868 control patients and 312 reconstructed patients. It accounted for the joint effect of prognostic factors (e.g., tumor size, nodal status, etc.) and, most importantly, the change in the hazard rate for recurrence resulting from specific events, such as reconstruction, occurring at varying time points. The multiple scale analysis provides evidence that following reconstruction women suffer a transitory, significant increase of recurrence risk during the first 2 years in comparison with not reconstructed patients (Fig. 8). This finding provides structural evidence for the enhancing effect of reconstructive surgery on subclinical metastases, which brings on the temporary raise of clinically evident recurrences. Such behavior is suggestive when it is considered in the light of the above-reported model. Indeed, the whole pattern would represents months since reconstruction/reference day. $Y$-axis represents the ratio between the six-month hazard for recurrence between reconstructed patients and controls

suggest that the early peak may be caused by an event that has been brought forward, and in the absence of reconstruction were to be expected at a later time. Taken together, the two analysis approaches provide evidence that reconstructed patients (a) suffer increased surgery-related recurrence rate following the usual bimodal pattern and (b) display disease-free survival that is not worse (maybe even better) than that of not reconstructed patients (Fig. 8).

Although not directly comparable, the recurrence pattern analysis apparently diverges somewhat from a previous investigation where trauma or intervening surgical procedures unrelated to cancer were not associated with an increased rate of breast cancer recurrence [42]. Still, the fact that the effect of delayed reconstruction on the recurrence dynamics does not translate into reduced recurrence-free or overall survival is in keeping with most reports on the same subject [28, 29, 40, 41, 43]. The reason underlying the similar or relatively better long-term outcome of reconstructed patients is presently undetermined. Patients opting for reconstruction tend to be younger and have less comorbidity. Most studies to date have focused on immediate reconstruction [43-45]. We are inclined to ascribe this finding to selection bias of patients receiving reconstruction due to factors here unaccounted for, such as socioeconomic conditions and better general health. The former factor is related to the finding that patients who are of lower socioeconomic status are more likely to have a recurrence than women of higher social class [46]. 
Furthermore, current smokers or diabetic patients are not accepted for microvascular free (DIEP) or pedicle flap surgery (TRAM). In addition, body mass index, which is a prognostic factor in cancer [47, 48], would not be higher than 30 in patients offered advanced reconstruction. Most patients undergoing DIEP or TRAM procedures have a secondary or even tertiary surgery performed due to cosmetic purposes or complications. Thus, the observations are measurements of the effect from the first extensive reconstructive surgical procedure, independent of the duration of reconstructive surgery or the number of surgical events. Again, such subsequent procedures might represent a possible bias and the observed effects might therefore be diluted.

In conclusion, our study indicates that reconstructive breast cancer surgery constitutes an independent stimulating event on the growth of micrometastases leading to accelerated relapse rates. The effect is similar to that observed after primary breast cancer surgery. Importantly, this does not translate into worse long-term disease-free survival. Our results may provide indirect evidence that immediate reconstruction would be more beneficial than delayed as this obviates one possible growth stimulating event. Still, randomized trials assessing this question are not ethically or practically feasible. Further studies are ongoing and will shed more light on tumor biological mechanisms behind the observed phenomenon.

Acknowledgments This work was partly supported by the University of Bergen and the Research Council of Norway through its Centres of Excellence funding scheme, project number 223250, and by grants from the Helse Vest Research Fund, The Italian Association for Cancer Research, and the Norwegian Cancer Society.

\section{Compliance with ethical standards}

Conflict of interest The authors declare no conflict of interests.

Open Access This article is distributed under the terms of the Creative Commons Attribution-NonCommercial 4.0 International License (http://creativecommons.org/licenses/by-nc/4.0/), which permits any noncommercial use, distribution, and reproduction in any medium, provided you give appropriate credit to the original author(s) and the source, provide a link to the Creative Commons license, and indicate if changes were made.

\section{References}

1. Brewster AM, Hortobagyi G, Broglio KR, Kau SW, Santa-Maria CA, Arun B, Buzdar AU, Booser DJ, Valero V, Bondy M, Esteva FJ (2008) Residual risk of breast cancer recurrence 5 years after adjuvant therapy. J Natl Cancer Inst 100(116): $1179-1183$

2. Fisher B (1980) Laboratory and clinical research in breast cancer: a personal adventure: the David A Karnofsky memorial lecture. Cancer Res 40:3863-3874
3. Demicheli R, Terenziani M, Valagussa P, Moliterni A, Zambetti M, Bonadonna G (1994) Local recurrences following mastectomy: support for the concept of tumor dormancy. J Natl Cancer Inst 86(1):45-48

4. Luzzi KJMI, Schmidt EE, Kerkvliet N, Morric VL, Chambers AF, Groom AC (1998) Multistep nature of metastatic inefficiency: dormancy of solitary cells after successful extravasation and limited survival of early micrometastases. Am J Pathol 153:865-873

5. Ghajar CMPH, Mori H, Matei IR, Evason KJ, Brazier H, Almeida D, Koller A, Hajjar KA, Stainier DYR, Chen EI, Lyden D, Bissell MJ (2013) The perivascular niche regulates breast tumour dormancy. Nat Cell Biol 15(7):807-817. doi:10.1038/ncb2767

6. Klauber-DeMore NVZ, LinkovI KJ, Brogen PI, Gerald WL (2001) Biological behavior of human breast cancer micrometastases. Clin Cancer Res 7:2434-2439

7. Aguirre-Ghiso JA (2007) Models, mechanisms and clinical evidence for cancer dormancy. Nat Rev Cancer 7:834-846

8. Naumov GAL, Folkman J (2006) Role of angiogenesis in human tumor dormancy: animal models of the angiogenic switch. Cell Cycle 5(16):1779-1787

9. Koebel CM, Vermi W, Swann JB, Zerafa N, Rodig SJ, Old LJ, Smyth MJ, Schreiber RD (2007) Adaptive immunity maintains occult cancer in an equilibrium state. Nature 450(7171):79037907

10. Malladi S, Macalinao DG, Jin X, He L, Basnet H, Zou Y, de Stanchina E, Massague J (2016) Metastatic latency and immune evasion through autocrine inhibition of WNT. Cell 165(1):45-60. doi:10.1016/j.cell.2016.02.025

11. Ranganathan AC, Adam A, Aguirre-Ghiso JA (2006) Opposing roles of mitogenic and stress signaling pathways in the induction of cancer dormancy. Cell Cycle 5(16):1799-1807

12. Sosa MSBP, Debnath J, Aguirre-Ghiso JA (2013) Regulation of tumor cell dormancy by tissue microenvironments and autophagy. Adv Exp Med Biol 734:773-789

13. Hanin LB-MS (2014) Reconstruction of the natural history of metastatic cancer and assessment of the effects of surgery: Gompertzian growth of the primary tumor. Math Biosci 247: 248-257

14. Demicheli RFM, Ambrogi F, Hiigins K, Boyd JA, Biganzoli E, Kelsey CR (2012) Recurrence dynamics for non-small-cell lung cancer, effect of surgery on the development of metastases. J Thorac Oncol 7:723-730

15. Kelsey CRFM, Ambrogi F, Higgins K, Boyd JA, Biganzoli E, Demicheli R (2013) Metastasis dynamics for non-small-cell lung cancer: effect of patient and tumor-related factors. Clin Lung Cancer 14(4):425-432

16. Fisher BGN, Saffer EA (1983) Influence of the interval between primary tumor removal and chemotherapy on kinetics and growth of metastases. Cancer Res 43:1488-1492

17. Peeters CF, Wobbes dWR T, Westphal JR, Ruers TJ (2006) Outgrowth of human liver metastases after resection of the primary colorectal tumor: a shift in the balance between apoptosis and proliferation. Int J Cancer 119(6):1249-1253

18. Curigliano GPJ, Bertolini F, Colleoni M, Peruzzotti G, de Braud F, Gandini S, Girlado A, Martella S, Orlando L, Munzone E, Pietri E, Luini A, Goldhirsch A (2005) Systemic effects of surgery: quantitative analysis of circulating basic fibroblast growth factor (bFGF), vascular endothelial growth factor (VEGF) and transforming growth factor beta (TGF- $\beta$ ) in patients with breast cancer who underwent limited or extended surgery. Breast Cancer Res Treat 93:35-40

19. Shao LOT, Sakamoo M, Mori S, Kodama T (2015) Activation of latent metastases in the lung after resection of a metastatic lymph node in a lymph node metastasis mouse model. Biochem Biophys Res Comm 460:543-548 
20. Dillekas H, Transeth M, Pilskog M, Assmus J, Straume O (2014) Differences in metastatic patterns in relation to time between primary surgery and first relapse from breast cancer suggest synchronized growth of dormant micrometastases. Breast Cancer Res Treat 146(143):627-736

21. Retsky MW, Demicheli R, Hrushesky WJ, Baum M, Gukas ID (2008) Dormancy and surgery-driven escape from dormancy help explain some clinical features of breast cancer. APMIS 116(117-118):730-741

22. Lee JW, Shahzad M, Lin YG, Armaiz-Pena G, Mangala LS, Han HD, Kim HS, Nam EJ, Jennings NB, Halder J, Nick AM, Stone RL, Lu C, Lutgendorf SK, Cole SW, Lokshin AE, Sood AK (2009) Surgical stress promotes tumor growth in ovarian carcinoma. Clin Cancer Res 15(18):2695-2702

23. Oosterling SJ, Van der Bij G, van Egmond M, van der Sijp JR (2005) Surgical trauma and peritoneal recurrence of colorectal carcinoma. Eur J Surg Oncol 31(31):29-37

24. Morihara K, Takenaka H, Morihara T, Kishimoto S (2007) Primary cutaneous anaplastic large cell lymphoma associated with vascular endothelial growth factor arising from a burn scar. J Am Acad Dermatol 57(55):S103-S105

25. Retsky M, Demicheli R, Hrushesky W, Baum M, Gukas I (2010) Surgery triggers outgrowth of latent distant disease in breast cancer: an inconvenient truth? Cancers (Basel) 30(32):305-337

26. Demicheli RRM, Swartzendruber DE, Bonadonna G (1997) Proposal for a new model of breast cancer metastatic development. Ann Oncol 8(11):1075-1080

27. Retsky MWDR, Swartzendruber DE, Bame PD, Wardwell RH, Bonadonna G, Speer JF, Valagussa P (1997) Computer simulation of a breast cancer metastasis model. Breast Cancer Res Treat 15(2):193-202

28. Isern AE, Manjer J, Malina J, Loman N, Mårtensson T, Bofin A, Hagen AI, Tengrup I, Landberg G, Ringberg A (2011) Risk of recurrence following delayed large flap reconstruction after mastectomy for breast cancer. Br J Surg 98(95):659-666

29. Hölmich LRDM, Foged Henriksen T, Krag C, Brix Tange U, Kjøller K, McLaughlin JK, Olsen JH, Friis S (2008) Delayed breast reconstruction with implants after invasive breast cancer does not impair prognosis. Ann Plast Surg 61:11-18

30. Registry TNC (2014) Nasjonalt kvalitetesregister for brystkreftÅrsrapport 2014. kvalitetsregistreno

31. Demicheli RAA, Miceli R, Valagussa P, Bonadonna G (1996) Time distribution of the recurrence risk for breast cancer patients undergoing mastectomy: further support about the concept of tumor dormancy. Breast Cancer Res Treat 41(2):177-185

32. Marubini E, Valsecchi MG (2004) Analysing survival data from clinical trials and observational studies. Wilay, Chichester

33. Ramlau-Hansen H (1983) Smoothing counting process intensities by means of Kernel functions. Ann Stat 11:453-466

34. Boracchi PBE, Marubini E (2003) Joint modelling of causespecific hazard functions with cubic splines: an application to a large series of breast cancer patients. Comput Statist Data Anal 42:243-262
35. Iacobelli SCB (2013) Multiple time scales in multi-state models. Stat Med 32:5315-5327

36. Demicheli RRM, Hrushesky WJM, Baum M, Gukas ID (2008) The effects of surgery on tumor growth: a century of investigations. Annal Oncol 19:1821-1828

37. Demicheli RRM, Hrushesky WJM, Baum M (2007) Tumor dormancy and surgery-driven dormancy interruption in breast cancer: learning from failures. Nat Clin Pract Oncol 4(12): 699-710

38. Antonio N, Bønnelykke-Behrndtz M, Ward L, Collin J, Christensen IJ, Steiniche T, Schmidt H, Feng Y, Martin P (2015) The wound inflammatory response exacerbates growth of pre-neoplastic cells and progression to cancer. EMBO J 34(17): 2219-2236

39. Voron T, Marcheteau E, Pernot S, Colussi O, Tartour E, Taieb J, Terme M (2014) Control of the immune response by pro-angiogenic factors. Front Oncol 4:70. doi:10.3389/fonc.2014.00070

40. Lindford AJ, Siponen E, Jahkola TA, Leidenius MH (2013) Effect of delayed autologous breast reconstruction on breast cancer recurrence and survival. World J Surg 37(12):2872-2882

41. Platt JBN, McLaughlin J, Semple JL (2015) Does breast reconstruction after mastectomy for breast cancer affect overall survival? Long-term follow-up of a retrospective population-based cohort. Plast Reconstr Surg 135(3):468e-476e

42. Allawi Z, Cuzick J, Baum M, ATAC/LATTE investigators (2012) Does trauma or an intercurrent surgical intervention lead to a short-term increase in breast cancer recurrence rates. Ann Oncol 23(4):866-869

43. Langstein HNCM-H, Singletary SE, Robb GL, Hoy E, Smith TL, Kroll SS (2003) Breast cancer recurrence after immediate reconstruction: patterns and significance. Plastic Reconstr Surg 11(2):712-720

44. Gieni M, Avram R, Dickson L, Farrokhyar F, Lovrics P, Faidi S, Sne N (2012) Local breast cancer recurrence after mastectomy and immediate breast reconstruction for invasive cancer: a metaanalysis. Breast 21(23):230-236

45. Eriksen CFJ, Wickman M, Lidbrink E, Krawiec K, Sandelin K (2011) Immediate reconstruction with implants in women with invasive breast cancer does not affect oncological safety in a matched cohort study. Breast Cancer Res Treat 127(2):439-446

46. Gordon NHCJ, Brumberg DJ, Berger NA (1992) Socioeconomic factors and race in breast cancer recurrence and survival. Am J Epidemiol 135(6):609-618

47. Chan DSNT (2015) Obesity and breast cancer: not only a risk factor of the disease. Curr Treat Options Oncol 16(5):22. doi:10. 1007/s11864-015-0341-9

48. Sinicrope FA, Foster N, Yothers G, Benson A, Seitz JF, Labianca R, Goldberg RM, Degramont A, O'Connell MJ, Sargent DJ, Adjuvant Colon Cancer Endpoints (ACCENT) Group (2013) Body mass index at diagnosis and survival among colon cancer patients enrolled in clinical trials of adjuvant chemotherapy. Cancer 119(8):1528-1536 